\title{
Stopping Power Calculations for Partially Stripped Projectiles in High Energy Region
}

\author{
M.Ç. Tufan, A. KÖroĞLu And H. GÜMÜß̧* \\ Department of Physics, Faculty of Sciences and Arts \\ Ondokuz Mayıs University, 55139 Samsun, Turkey
}

(Received July 30, 2004; revised version November 15, 2004)

The electronic stopping cross-section is calculated in the spirit of the Bethe theory. Interaction potential between projectile and target is regarded to have a Coulombic character and we have modified it to take into account velocity dependences on a number of bound electrons of projectile and an effective charge of projectile and target. These velocity dependences are obtained from the Bohr adiabatic criterion using the Thomas-Fermi atomic model. We have get the electronic stopping cross-section expression using the Bethe approximation; we obtained the stopping cross-section of $\mathrm{C}$ and $\mathrm{Al}$ for $\mathrm{C}, \mathrm{O}$, and $\mathrm{Si}$ ions from this expression and compared our results with experiment and other theoretical calculations.

PACS numbers: $34.50 . \mathrm{Bw}, 34.10 .+\mathrm{x}$

\section{Introduction}

In the interaction between charged particles and solids, the quantity of stopping power or the average energy loss per unit path length plays an important role in many fields such as impurity atom implantation in producing semiconductor devices, structure analysis of solid target by Rutherford backscattering spectroscopy (RBS), and plasma-first wall interactions in nuclear-fusion reactor. Therefore, the electronic stopping power of matter for energetic ions has been studied in ion-solid and ion-gas interactions. The studies of the electronic stopping power have begun at the beginning of the last century [1]. Experimental data compilations of

*corresponding author; e-mail: hgumus@omu.edu.tr 
electronic energy loss have been performed $[2,3]$. Furthermore, theoretical formulations of electronic stopping power have been carried out extensively with some approximations [4-10], and some scientists, such as Bates and Griffing [11], Brandt and Kitagawa [12], Kim and Cheng [13], and Cabrera-Trujillo et al. [14] have studied this problem in the essence of former theories in order to obtain better results and to develop new theories.

Using the Bethe theory of stopping, various efforts have been performed to account for the stopping of partially striped ions. For this purpose, Kim and Cheng [13] suggested that in the original Bethe formula, the effective projectile nuclear charge $Z_{\text {eff }}$ and the target mean ionization energy $I_{\text {eff }}$ can be used instead of $Z_{1}$ and ${ }_{2} I_{0}$, respectively. Moreover, Cabrera-Trujillo et al. [14] have reported a Bethe-like theory for partially stripped projectiles carrying a velocity-dependent number of bound electrons in the first Born approximation. In our work, we consider not only partially stripped projectiles carrying the velocity-dependent number of bound electrons, but also the target and projectile effective charge.

In Sec. 2 we develop the stopping cross-section in the first Born approximation by considering both projectile and target electronic structures. In Sec. 3 we perform a number of bound electrons of projectile, and effective charge of target, and effective mean excitation energies of projectile and target, depending on projectile velocity. A final expression of the electronic stopping cross-section is obtained in Sec. 4 using the results of Secs. 2 and 3. We compare our results with other theoretical and experimental results in Sec. 5, and our conclusions are presented in Sec. 6 .

\section{Stopping cross-section expression in the first Born approximation}

In the laboratory frame, the stopping cross-section for ion with velocity $v$ colliding with stationary target is given by [14]

$$
S_{\mathrm{e}}(v)=\sum_{n, m} \int \frac{\mathrm{d} \sigma_{n, m}}{\mathrm{~d} \Omega}\left[\left(E_{n}-E_{n_{0}}\right)+\left(E_{m}-E_{m_{0}}\right)\right] \mathrm{d} \Omega,
$$

where $E_{n 0}$ and $E_{m 0}$, and $E_{n}$ and $E_{m}$ are projectile and target energies before and after the collision, respectively, and $\mathrm{d} \sigma_{n, m} / \mathrm{d} \Omega$ is the differential cross-section. In the first Born approximation, the differential cross-section for nonrelativistic velocities of projectile is calculated by

$$
\frac{\mathrm{d} \sigma_{n, m}}{\mathrm{~d} \Omega}=\frac{M_{1}^{2}}{(2 \pi)^{2} \hbar^{4}} \frac{k}{k_{0}}\left|\left\langle n m k|V| n_{0} m_{0} k_{0}\right\rangle\right|^{2},
$$

where $V$ is the interaction potential between projectile and target [15].

In order to obtain the differential cross-section, first we must determine the interaction potential. As the interaction between projectile and target is Coulombic, we can express the interaction potential as 


$$
\begin{aligned}
& V(\boldsymbol{x}, \boldsymbol{y}, \boldsymbol{R})=\frac{Z_{1} Z_{2} e^{2}}{\boldsymbol{R}}-\sum_{i=1}^{Z_{2}^{*}} \frac{Z_{1} e^{2}}{\left|\boldsymbol{R}-\boldsymbol{y}_{i}\right|}-\sum_{i=1}^{N_{1}} \frac{Z_{2} e^{2}}{\left|\boldsymbol{R}+\boldsymbol{x}_{i}\right|} \\
& +\sum_{i, j=1}^{N_{1}, Z_{2}^{*}} \frac{e^{2}}{\left|\boldsymbol{y}_{j}-\boldsymbol{R}-\boldsymbol{x}_{i}\right|},
\end{aligned}
$$

where $N_{1}, Z_{1}$, and $Z_{2}^{*}$, are the number of bound electrons of projectile, nuclear charge of projectile and target, and the number of electrons of target interacting with projectile (or effective charge of target), respectively. $\boldsymbol{R}, \boldsymbol{x}_{i}, \boldsymbol{y}_{j}$ are projectile's position relative to the center of mass of target, the position vector of electron $i$ of the projectile with respect to the center of mass of projectile, and the position vector of electron $j$ of the target with respect to the center of mass of target, respectively.

Substituting Eqs. (2) and (3) into Eq. (1), one can obtain

$$
\begin{aligned}
S_{\mathrm{e}}(v) & =\frac{2 e^{4}}{m_{\mathrm{e}} v^{2}} \sum_{\mathrm{n}, \mathrm{m}}\left\{\int_{\mathrm{q}_{\min }}^{\mathrm{q}_{\max }}\left|\mathrm{Z}_{2} \delta_{\mathrm{m}_{0} \mathrm{~m}}-{ }_{2} \mathrm{M}_{\mathrm{m}_{0} \mathrm{~m}}(\boldsymbol{q})\right|^{2}{ }_{1} \mathrm{~F}_{\mathrm{n}_{0} \mathrm{n}}(\boldsymbol{q}) \frac{\mathrm{d} \boldsymbol{q}}{\mathrm{q}^{2}}\right. \\
& \left.+\int_{q_{\min }}^{q_{\max }}\left|Z_{1} \delta_{n_{0} n}-{ }_{1} M_{n_{0} n}(\boldsymbol{q})\right|^{2}{ }_{2} F_{m_{0} m}(\boldsymbol{q}) \frac{\mathrm{d} \boldsymbol{q}}{q^{2}}\right\} \\
& =S_{\mathrm{e}, 1}(v)+S_{\mathrm{e}, 2}(v),
\end{aligned}
$$

where

$$
\begin{aligned}
& { }_{1} M_{n_{0} n}(\boldsymbol{q})=\left\langle n_{0}\left|\sum_{j=1}^{N_{1}} \mathrm{e}^{-\mathrm{i} \boldsymbol{q} \boldsymbol{\boldsymbol { x } _ { j }}}\right| n\right\rangle, \\
& { }_{2} M_{m_{0} m}(\boldsymbol{q})=\left\langle m_{0}\left|\sum_{j=1}^{Z_{2}^{*}} \mathrm{e}^{-\mathrm{i} \boldsymbol{q} \boldsymbol{y}_{j}}\right| m\right\rangle
\end{aligned}
$$

are the atomic form factors $[1,16]$ of the transition between the initial state and final state of projectile and target, respectively, where we used the definition $\boldsymbol{q}=\boldsymbol{k}-\boldsymbol{k}_{0}$ to write $\mathrm{d} \Omega=q \mathrm{~d} q \mathrm{~d} \varphi / k k_{0}=\mathrm{d} \boldsymbol{q} / k k_{0}$. The generalized oscillators strengths (GOS) of projectile and target [4] are given by

$$
\begin{aligned}
& { }_{1} F_{n_{0} n}(\boldsymbol{q})=\left.\left.\frac{2 m_{\mathrm{e}}}{\hbar q^{2}}\right|_{1} M_{n_{0} n}(\boldsymbol{q})\right|^{2} w_{n n_{0}}, \\
& { }_{2} F_{m_{0} m}(\boldsymbol{q})=\left.\left.\frac{2 m_{\mathrm{e}}}{\hbar q^{2}}\right|_{2} M_{m_{0} m}(\boldsymbol{q})\right|^{2} w_{m m_{0}} .
\end{aligned}
$$

If one uses the Bethe sum rule [4] and Hartree-Fock atomic wave functions, then the stopping cross-section becomes 


$$
\begin{aligned}
S_{\mathrm{e}}(v) & =\frac{2 e^{4}}{m_{\mathrm{e}} v^{2}} \int_{\mathrm{q}_{\min }}^{\mathrm{q}_{\max }}\left\{\mathrm{Z}_{2}^{*}\left[\mathrm{Z}_{1}-{ }_{1} \mathrm{M}_{\mathrm{n}_{0} \mathrm{n}_{0}}(\boldsymbol{q})\right]^{2}\right. \\
+ & \left.N_{1}\left[Z_{2}-{ }_{2} M_{m_{0} m_{0}}(\boldsymbol{q})\right]^{2}\right\} \frac{\mathrm{d} \boldsymbol{q}}{q^{2}} .
\end{aligned}
$$
given as

$$
q_{\min }=\frac{w_{n n_{0}}+w_{m m_{0}}}{v}, \quad q_{\max }=\frac{2 m_{\mathrm{e}} v}{\hbar},
$$

respectively. Cabrera-Trujillo et al. [14] have given an applicable average value for $q_{\mathrm{min}}$, which is independent of the excited state of the system:

$$
q_{\min _{\mathrm{AV}}}=\frac{\left(w_{n n_{0}}+w_{m m_{0}}\right)_{\mathrm{AV}}}{v}=\frac{\varepsilon}{\hbar v} \text {. }
$$

Following the procedure in Ref. [14], but using our considerations, we have obtained $\varepsilon$ as

$$
\varepsilon={ }_{2} I_{0}^{* \frac{1}{1+\alpha}}{ }_{1} I_{0}^{*} \frac{z_{1}^{*}}{N_{1}} \frac{\alpha}{1+\alpha},
$$

where

$$
\alpha=\frac{\left(Z_{2}-Z_{2}^{*}\right)^{2} N_{1}}{\left(Z_{1}-N_{1}\right)^{2} Z_{2}^{*}}
$$

\section{Bohr adiabatic criterion}

\subsection{Determination of $N_{1}(v)$ and $Z_{2}^{*}(v)$}

In this study, a modification to the Bethe theory of stopping power has been suggested for a projectile with bound electrons and for target electrons interacting with bound electrons of projectile. It is necessary to know the number of electrons bound to the projectile $N_{1}$ and the effective charge of target $Z_{2}^{*}$ to apply this theory.

Generally, the calculation of an equilibrium distribution of electrons in the projectile needs detailed estimates of the cross-sections for electron capture and loss. It is therefore more convenient to use the Bohr adiabatic criterion [18]. This criterion states that these electrons are stripped from the projectile if orbital velocity of electrons, $v_{\mathrm{e}}$, is less than the projectile velocity, otherwise are kept since the collision is adiabatic. This criterion [19] can be given by

$$
v=b v_{\mathrm{F}}\left(r_{\mathrm{c}}\right) \text {, }
$$

where $v_{\mathrm{F}}(r)=\frac{\hbar}{m_{\mathrm{e}}}\left[3 \pi^{2} \rho(r)\right]^{1 / 3}$ is the Fermi velocity, $\rho(r)$ is the electron density, $b$ is the proportional constant of the order of 1.26 , and $r_{\mathrm{c}}$ is the distance of an electron bound to the projectile from the nucleus. The velocity of the electron is 
equal to the velocity of the projectile $\left(v_{\mathrm{e}}=v\right)$ at $r_{\mathrm{c}}$ and an electron is stripped from the projectile at $r_{\mathrm{c}}<r$ since the velocity of electron is less than the velocity of the projectile. Thus, the number of electrons bound to the projectile is given by

$$
N_{1}(v)=\int_{0}^{r_{c}} 4 \pi r^{2} \rho_{1}(r) \mathrm{d} r .
$$

In order to determine $r_{\mathrm{c}}$ and thus $N_{1}(v)$, we use the Thomas-Fermi theory (TF) [20], which describes an ion with $N_{1}$ bound electrons by radially symmetric electron density

$$
\rho_{1}(r)=\frac{N_{1}}{4 \pi \Lambda_{1}^{2} r} \frac{\mathrm{d}^{2} \Phi(x)}{\mathrm{d} x^{2}},
$$

where $x=r / \Lambda_{1}, \Phi(x)$ is the screening function obtained from the solution of TF equation, and the screening length, $\Lambda_{1}$, is a variational parameter and obtained by minimizing the total energy,

$$
E=E_{\mathrm{ne}}+\lambda E_{\mathrm{ee}}+E_{\mathrm{kin}},
$$

as a function of the number of electron $N_{1}(v)$. Here, the first term is the interaction between nucleus and electrons, the second term is the interaction between electrons, where $\lambda$ is a parameter including both correlation and exchange [12] and the last term is the kinetic energy of electrons. The total energy must satisfy the conditions given below:

$$
\frac{\partial E}{\partial \Lambda_{1}}=0,\left.\quad \frac{\partial E}{\partial N_{1}}\right|_{Z_{1}}=0,
$$

where the second condition states that a neutral atom has a lower energy than its ions.

In this study, we used the screening function, $\Phi(x)$, defined in Ref. [21]:

$$
\begin{aligned}
& \Phi(x)=0.1818 \exp (-3.2 x)+0.5099 \exp (-0.9423 x) \\
& \quad+0.2802 \exp (-0.4028 x)+0.02817 \exp (-0.2016 x) .
\end{aligned}
$$

Replacing Eq. (17) into Eq. (14), and following a standard procedure [22] for calculation of the total energy, we obtain

$$
E=-\frac{Z_{1}^{7 / 3}}{c}\left(\frac{N_{1}}{Z_{1}}\right)^{1 / 3}\left(1-\frac{1}{7} \frac{N_{1}}{Z_{1}}\right)^{2} \frac{e^{2}}{a_{0}},
$$

where $c=0.9660342260$. Also for the screening length, we have

$$
\Lambda_{1}=0.570338107 \frac{a_{0}}{Z_{1}^{1 / 3}\left(1-\frac{1}{7} \frac{N_{1}}{Z_{1}}\right)}\left(\frac{N_{1}}{Z_{1}}\right)^{2 / 3},
$$

varying with a number of bound electrons and 


$$
\Lambda_{1}=0.665394458 a_{0} Z_{1}^{-1 / 3}
$$

depending on atomic number.

In order to calculate the critical length from the Bohr stripping criteria, we must use the electronic charge density

$$
\rho_{1}(x)=\frac{Z_{1}}{4 \pi \Lambda_{1}^{3}} \frac{F(x)}{x},
$$

where $F(x)$ is the second derivatives of the screening function with respect to $x$, $\left(\frac{\mathrm{d}^{2} \Phi(x)}{\mathrm{d} x^{2}}\right)$. Substituting Eq. (20) into Eq. (12), we obtain as

$$
\left[F\left(x_{\mathrm{c}}\right)\right]^{1 / 3} k-v x_{\mathrm{c}}^{1 / 3}=0,
$$

where

$$
k=b\left(\frac{3 \pi}{4}\right)^{1 / 3} \frac{Z_{1}^{2 / 3}}{0.665394458} .
$$

The stripping length is numerically calculated from the solution of Eq. (21).

All expressions were derived for projectiles up to now, but similar expressions can be derived for targets. Furthermore, $Z_{2}^{*}(v)$ can be expressed as [23]

$$
Z_{2}^{*}(v)=\int_{r_{\mathrm{c}}}^{\infty} 4 \pi r^{2} \rho_{2}(r) \mathrm{d} r .
$$

\subsection{The calculation of mean excitation energy}

The mean excitation energy is given by [17]

$$
\ln I=\frac{1}{Z} \sum_{n} f_{n 0} \ln \left(E_{n}-E_{0}\right),
$$

where $Z$ is the atomic number and $f_{n 0}$ is optical dipole oscillator strengths corresponding to the excitation energy, $E_{n}-E_{0}$. Lindhard and Scharff proposed a statistical approach to the mean excitation energy in the energy loss problem [24]:

$$
\ln I=\frac{1}{Z} \int_{0}^{\infty} \ln \left[\gamma \hbar w_{\mathrm{p}}(r)\right] 4 \pi r^{2} \rho(r) \mathrm{d} r,
$$

where $r$ is the distance from nucleus, $\rho(r)$ is the electronic charge density between $r$ and $r+\mathrm{d} r, w_{\mathrm{p}}$ is the plasma frequency, $w_{\mathrm{p}}(r)=\left[4 \pi e^{2} \rho(r) / m_{\mathrm{e}}\right]^{1 / 2}$ and $\gamma$ is a constant estimated by Lindhard and Scharff to be $\sqrt{2}$. Furthermore, this constant value can be calculated by considering Eq. (25) equal to an experimental value of the mean excitation energy [23].

The mean excitation energy is a quantity varying with velocity in reality and can be calculated by [23]

$$
\ln I^{*}=\frac{1}{Z^{*}} \int_{r_{\mathrm{c}}}^{\infty} \ln \left[\gamma \hbar w_{\mathrm{p}}(r)\right] 4 \pi r^{2} \rho(r) \mathrm{d} r .
$$


If the electronic charge density calculated by using the method of ThomasFermi for neutral atom and plasma frequency are inserted into Eq. (26), the effective mean excitation energy of projectile and target are obtained as (in $[\mathrm{eV}]$ )

$$
{ }_{i} I^{*}=27.2 \gamma Z_{i} c^{-3 / 2} \exp (\alpha)
$$

where $\alpha$ is given by

$$
\alpha=\frac{Z_{i}}{2 Z_{i}^{*}} \int_{x_{\mathrm{c}}}^{\infty} \ln \left[\frac{F(x)}{x}\right] F(x) x \mathrm{~d} x,
$$

and solved numerically after $x_{\mathrm{c}}$ is found.

\section{The calculation of stopping cross-section}

We also need to know the atomic form factors for both projectile and target to calculate the stopping cross-section, $S_{\mathrm{e}}(v)$ in the first Born approximation. Assuming that the ground states of both projectile and target are of spherical symmetry, Eq. (5) becomes [13]

$$
{ }_{i} M_{00}(q)=4 \pi \int \frac{\rho_{i}(r) \sin (q r)}{q r} r^{2} \mathrm{~d} r, \quad i=1,2 .
$$

With respect to the dimensionless variable $x$, Eq. (29) becomes

$$
{ }_{i} M_{00}(q)=4 \pi \Lambda_{i}^{2} \int \frac{\rho_{i}(x) \sin \left(q \Lambda_{i} x\right)}{q} x \mathrm{~d} x,
$$

where $\rho_{i}$ is the electronic charge density.

Recalling $\rho_{i}$ 's in Eq. (30) and integrating with respect to $x$, the atomic form factor becomes

$$
\begin{gathered}
{ }_{1} M_{00}(q)=N_{1}\left(0.5996098+2.3609939 q^{6} \Lambda_{1}^{6}+7.2794472 q^{4} \Lambda_{1}^{4}+1.7376768 q^{2} \Lambda_{1}^{2}\right) \\
\times\left[\left(q^{2} \Lambda_{1}^{2}+10.24\right)\left(q^{2} \Lambda_{1}^{2}+0.88793\right)\left(q^{2} \Lambda_{1}^{2}+0.16225\right)\left(q^{2} \Lambda_{1}^{2}+0.04064\right)\right]^{-1}
\end{gathered}
$$

for projectiles and also

$$
\begin{gathered}
{ }_{2} M_{00}(q)=Z_{2}^{*}\left(0.5996098+2.3609939 q^{6} \Lambda_{2}^{6}+7.2794472 q^{4} \Lambda_{2}^{4}+1.7376768 q^{2} \Lambda_{2}^{2}\right) \\
\times\left[\left(q^{2} \Lambda_{2}^{2}+10.24\right)\left(q^{2} \Lambda_{2}^{2}+0.88793\right)\left(q^{2} \Lambda_{2}^{2}+0.16225\right)\left(q^{2} \Lambda_{2}^{2}+0.04064\right)\right]^{-1}
\end{gathered}
$$

for a target.

Now, substituting Eqs. (31) and (32) into Eq. (7), we find that the contributions to the stopping cross-section from the electronic structure of the target become

$$
\begin{aligned}
S_{\mathrm{e}, 2} & =\frac{4 \pi e^{4}}{m_{\mathrm{e}} v^{2}} Z_{2}^{*} Z_{1}^{2}\left\{i_{\mathrm{f}}^{2} \ln \left(\frac{q_{\max }}{\bar{q}_{\min }}\right)\right. \\
& +\left(1-i_{\mathrm{f}}\right)\left[0.0068837\left(i_{\mathrm{f}}+25.41\right) \ln \left(\frac{\left(q_{\max } \Lambda_{1}\right)^{2}+10.24}{\left(\bar{q}_{\min } \Lambda_{1}\right)^{2}+10.24}\right)\right.
\end{aligned}
$$




$$
\begin{aligned}
& +0.19887\left(i_{\mathrm{f}}+1.564\right) \ln \left(\frac{\left(q_{\max } \Lambda_{1}\right)^{2}+0.88793}{\left(\bar{q}_{\min } \Lambda_{1}\right)^{2}+0.88793}\right) \\
& +0.26320\left(i_{\mathrm{f}}+0.064605\right) \ln \left(\frac{\left(q_{\max } \Lambda_{1}\right)^{2}+0.16225}{\left(\bar{q}_{\min } \Lambda_{1}\right)^{2}+0.16225}\right) \\
& \left.+0.031123\left(i_{\mathrm{f}}-0.094872\right) \ln \left(\frac{\left(q_{\max } \Lambda_{1}\right)^{2}+0.040643}{\left(\bar{q}_{\min } \Lambda_{1}\right)^{2}+0.040643}\right)\right] \\
& +\left(1-i_{\mathrm{f}}\right)^{2}\left[0.16922\left(\frac{1}{\left(q_{\max } \Lambda_{1}\right)^{2}+10.24}-\frac{1}{\left(\bar{q}_{\min } \Lambda_{1}\right)^{2}+10.24}\right)\right. \\
& +0.11543\left(\frac{1}{\left(q_{\max } \Lambda_{1}\right)^{2}+0.88793}-\frac{1}{\left(\bar{q}_{\min } \Lambda_{1}\right)^{2}+0.88793}\right) \\
& +0.0063692\left(\frac{1}{\left(q_{\max } \Lambda_{1}\right)^{2}+0.16225}-\frac{1}{\left(\bar{q}_{\min } \Lambda_{1}\right)^{2}+0.16225}\right) \\
& \left.\left.+0.000016126\left(\frac{1}{\left(q_{\max } \Lambda_{1}\right)^{2}+0.040643}-\frac{1}{\left(\bar{q}_{\min } \Lambda_{1}\right)^{2}+0.040643}\right)\right]\right\}
\end{aligned}
$$

and similarly, contributions to the stopping cross-section from the electronic structure of the projectile become

$$
\begin{aligned}
S_{\mathrm{e}, 1} & =\frac{4 \pi e^{4}}{m_{\mathrm{e}} v^{2}} Z_{1}\left(1-i_{\mathrm{f}}\right) Z_{2}^{2}\left\{\left(1-\frac{Z_{2}^{*}}{Z_{2}}\right)^{2} \ln \left(\frac{q_{\max }}{\bar{q}_{\min }}\right)\right. \\
& +\left(\frac{Z_{2}^{*}}{Z_{2}}\right)\left[0.0068837\left(26.41-\frac{Z_{2}^{*}}{Z_{2}}\right) \ln \left(\frac{\left(q_{\max } \Lambda_{2}\right)^{2}+10.24}{\left(\bar{q}_{\min } \Lambda_{2}\right)^{2}+10.24}\right)\right. \\
& +0.19887\left(2.564-\frac{Z_{2}^{*}}{Z_{2}}\right) \ln \left(\frac{\left(q_{\max } \Lambda_{2}\right)^{2}+0.88793}{\left(\bar{q}_{\min } \Lambda_{2}\right)^{2}+0.88793}\right) \\
& +0.26320\left(1.064605-\frac{Z_{2}^{*}}{Z_{2}}\right) \ln \left(\frac{\left(q_{\max } \Lambda_{2}\right)^{2}+0.16225}{\left(\bar{q}_{\min } \Lambda_{2}\right)^{2}+0.16225}\right) \\
& \left.+0.031123\left(0.905128-\frac{Z_{2}^{*}}{Z_{2}}\right) \ln \left(\frac{\left(q_{\max } \Lambda_{2}\right)^{2}+0.040643}{\left(\bar{q}_{\min } \Lambda_{2}\right)^{2}+0.040643}\right)\right] \\
& +\left(\frac{Z_{2}^{*}}{Z_{2}}\right)\left[0.16922\left(\frac{1}{\left(q_{\max } \Lambda_{2}\right)^{2}+10.24}-\frac{1}{\left(\bar{q}_{\min } \Lambda_{2}\right)^{2}+10.24}\right)\right. \\
& +0.11543\left(\frac{1}{\left(q_{\max } \Lambda_{2}\right)^{2}+0.88793}-\frac{1}{\left(\bar{q}_{\min } \Lambda_{2}\right)^{2}+0.88793}\right) \\
+ & 0.0063692\left(\frac{1}{\left(q_{\max } \Lambda_{2}\right)^{2}+0.16225}-\frac{1}{\left(\bar{q}_{\min } \Lambda_{2}\right)^{2}+0.16225}\right)
\end{aligned}
$$




$$
\left.\left.+0.000016126\left(\frac{1}{\left(q_{\max } \Lambda_{2}\right)^{2}+0.040643}-\frac{1}{\left(\bar{q}_{\min } \Lambda_{2}\right)^{2}+0.040643}\right)\right]\right\},
$$

where $i_{\mathrm{f}}$ is the ionization fraction [12] and defined as

$$
i_{\mathrm{f}}=1-\frac{N_{1}}{Z_{1}} .
$$

\section{Results and discussion}

In this study, the stopping cross-section has been calculated by considering the velocity dependent charge state of both projectile and target. When the velocity of projectile is increased, a number of bound electrons of projectile, $N_{1}$, are decreasing and become zero at rather high velocities, $N_{1} \rightarrow 0$ or $N_{1} / Z_{1} \rightarrow 0$. However, both projectile and target effective charges become larger, $Z_{1}^{*} \rightarrow Z_{1}$ and $Z_{2}^{*} \rightarrow Z_{2}$. Figure 1 shows this variation with respect to the effective ion velocity,

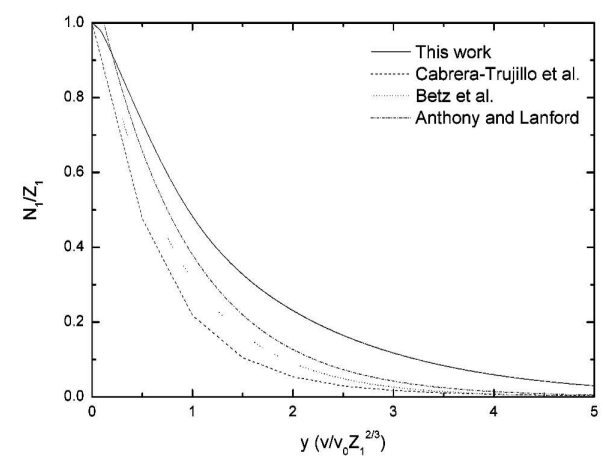

Fig. 1. Fraction of electrons bound to the projectile versus velocity of projectile, - this work; - - Cabrera-Trujillo et al. [14]; ․ Betz et al. [25]; - - - Anthony and Lanford [26].

$y(v)=v /\left(v_{0} Z_{1}^{2 / 3}\right)$. In addition, at high velocities the effective mean excitation energy of both projectile and target becomes a mean excitation energy of their neutral atoms, i.e. $\alpha=0$ and $\varepsilon={ }_{2} I_{0}$. Therefore, the stopping cross-section becomes

$$
S_{\mathrm{e}}(v)=\frac{4 \pi e^{4}}{m_{\mathrm{e}} v^{2}} Z_{1}^{2} Z_{2} \ln \left(\frac{2 m_{\mathrm{e}} v^{2}}{{ }_{2} I_{0}}\right) .
$$

That is just a standard Bethe result in the first Born approximation.

Figure 2 shows total energy of ion versus ionization fraction. As seen from Fig. 2, our calculation agrees with previous calculations $[12,14]$. Hence, the electronic charge density we are used performs well, and the calculation of bound electrons and of effective charge is sufficiently accurate. 


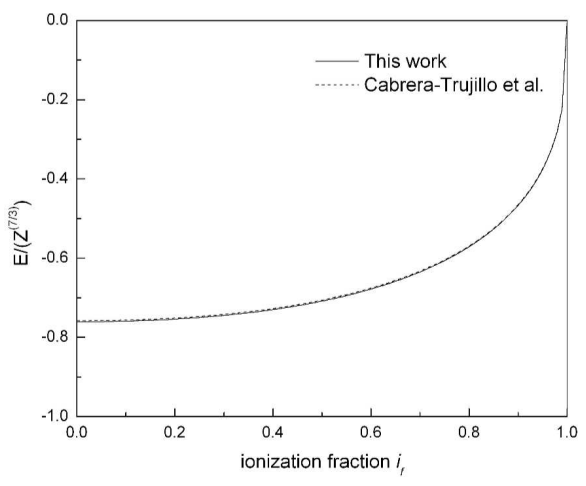

Fig. 2. Total energy of the ion as a function of the ionization fraction, $i_{\mathrm{f}}$; - this work; - Cabrera-Trujillo et al. [14].

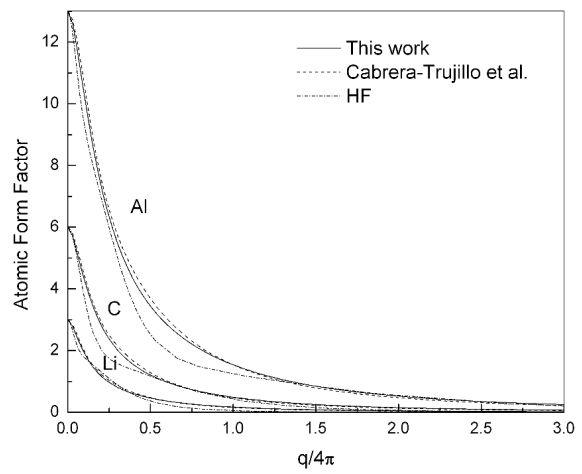

Fig. 3. Comparison of the form factor for neutral atoms as a function of the transfer moment $q$; - this work; - - Cabrera-Trujillo et al. [14]; - - - HF calculations [27].

In Fig. 3 the obtained results of the atomic form factors for several neutral atoms from calculations of our and of Ref. [14] are compared with HF results, calculated by Hubbel et al. [27]. As seen, our results agree with HF better than in Ref. [14]. In the calculations of the atomic form factor, a difference between our method and the method of Cabrera-Trujillo et al. is a screening function. Figure 3 shows this difference.

In order to obtain the total stopping cross-section, contributions of electronic structure of projectile and target to the total stopping cross-section must be added:

$$
S_{\mathrm{e}, \mathrm{T}}=S_{\mathrm{e}, 1}+S_{\mathrm{e}, 2}
$$

To calculate the total stopping cross-section, first the stripping length, $x_{\mathrm{c}}$, is calculated numerically from Eq. (21) and then using this value $Z_{i}^{*}$ and ${ }_{i} I^{*}$ are obtained from Eq. (23) and (27), respectively. $N_{i}$ is calculated from

$$
N_{i}=Z_{i}-Z_{i}^{*}
$$


After the above values are obtained, the total stopping cross-section is easily calculated from Eqs. (33) and (34). We calculated the total stopping cross-section of $\mathrm{C}, \mathrm{O}$, and $\mathrm{Si}$ on $\mathrm{C}$ and $\mathrm{Al}$, and we show it in Figs. 4a and b, 5a and b, and $6 \mathrm{a}$ and $\mathrm{b}$, respectively. In all of the figures, $-, \square, \cdots, \times, \nabla$, and $\bigcirc$ represent the results of this work, Hubert et al. [3], Cabrera-Trujillo et al. [14], Bethe [4], Paul and Schinner [28], and SRIM 2003 [29], respectively. As seen, our results agree with experimental and theoretical results in the range of the Bethe condition, $v>v_{0}$, are fulfilled. On the other hand, at the lower ion energies our results are better than the ones of Cabrera-Trujillo et al. [14].

In Fig. 4a and b, the stopping cross-sections of $\mathrm{C}$ and $\mathrm{Al}$ for $\mathrm{C}$ ions are showed, respectively. For the stopping power of $\mathrm{C}$ and $\mathrm{Al}$, at the energy range of 1-20 MeV/nucl., our results agree within $9 \%$ and $17 \%$ with Ref. [28], while the results from the calculations of Cabrera-Trujillo et al. [14] agree within $9 \%$ and $10 \%$, respectively. For $\mathrm{C}$ target, at the energy range of $1-2.5 \mathrm{MeV} / \mathrm{nucl}$, the agreement with experimental results [28] is $13 \%$ for this work and $21 \%$ for
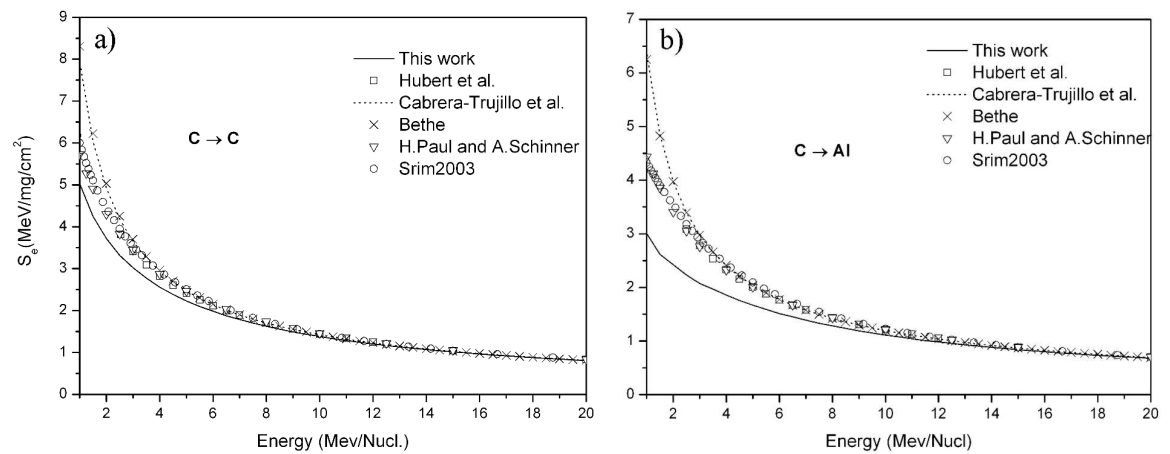

Fig. 4. The calculated electronic stopping cross-section of (a) $\mathrm{C}$ on $\mathrm{C}$ and (b) $\mathrm{C}$ on $\mathrm{Al}$ as a function of projectile velocity is compared with experimental and theoretical results.
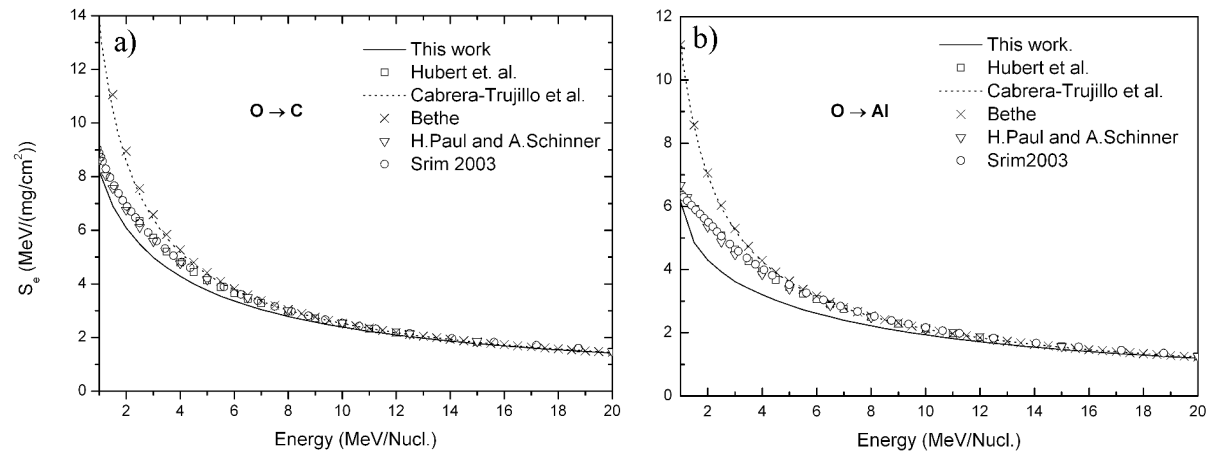

Fig. 5. The calculated electronic stopping cross-section of (a) $\mathrm{O}$ on $\mathrm{C}$ and (b) $\mathrm{O}$ on $\mathrm{Al}$ as a function of projectile velocity is compared with experimental and theoretical results. 

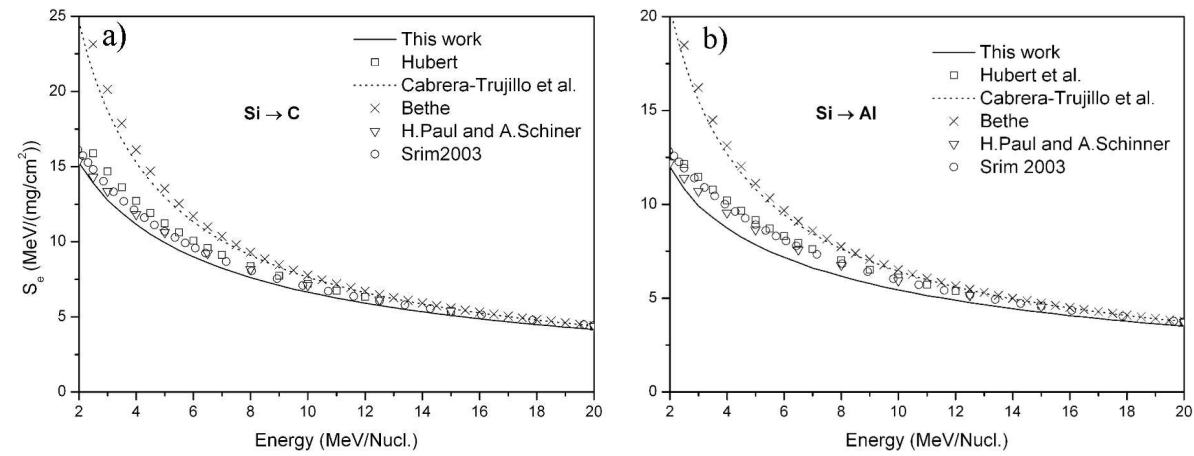

Fig. 6. The calculated electronic stopping cross-section of (a) Si on C and (b) Si on $\mathrm{Al}$ as a function of projectile velocity is compared with experimental and theoretical results.

Ref. [14], but for Al target at the same energy range, the agreement with Ref. [28] is approximately $30 \%$ for this work and $33 \%$ for Ref. [14]. On the other hand, comparison with other theoretical calculations [3, 29] is seen in Fig. 4a and b.

Figure $5 \mathrm{a}$ and $\mathrm{b}$ shows the stopping cross-section of $\mathrm{O}$ ions in $\mathrm{C}$ and $\mathrm{Al}$ target, respectively. For our results, agreements with the experimental results at the energy range of $1-20 \mathrm{MeV} /$ nucl. are $8 \%$ for $\mathrm{C}$ target and $13 \%$ for $\mathrm{Al}$ target, while the results of Ref. [14] agree within $14 \%$ and $16 \%$ for $\mathrm{C}$ and $\mathrm{Al}$ targets, respectively. Below $2.5 \mathrm{MeV} /$ nucl., the agreements of our results are more satisfactory than other calculations $[4,14]$.

Figure $6 \mathrm{a}$ and $\mathrm{b}$ indicates the stopping cross-sections of $\mathrm{C}$ and $\mathrm{Al}$ targets for incident $\mathrm{Si}$ ions. Like the previous figures, this figure shows the same behavior. But here, the agreement with experimental results is more accurate, i.e. our results agree within $5 \%$ and $9 \%$ with experimental results [28], while the results from the calculations of Cabrera-Trujillo et al. [14] agree within $33 \%$ and $38 \%$, for $\mathrm{C}$ and $\mathrm{Al}$ targets, respectively.

\section{Conclusions}

In this work, we derive the electronic stopping power by taking the Bethe approximation as a basis. In contrast to the Bethe theory [4] and other approximations [13, 14], our calculations of the electronic stopping cross-section depend on velocity dependent projectile and target electronic structure. Since our calculations of velocity dependence have been performed using TF atomic theory and other statistical procedures for the numerical calculations, our results for the projectile-target system with high $Z$ are better than the ones for low $Z$. Our results agree with experimental results within $5-9 \%$ for $\mathrm{C}-\mathrm{C}, \mathrm{O}-\mathrm{C}, \mathrm{Si}-\mathrm{C}$, and $\mathrm{Si}-\mathrm{Al}$ systems, and $12-17 \%$ for $\mathrm{C}-\mathrm{Al}$, and $\mathrm{O}-\mathrm{Al}$ systems. 


\section{References}

[1] U. Fano, A.R.P. Rau, Atomic Collisions and Spectra, Academic Press, New York 1986.

[2] J.F. Janni, At. Data Nucl. Data Tables 27, 341 (1982).

[3] F. Hubert, R. Bimbot, H. Gauvin, At. Data. Nucl. Data Tables 46, 1 (1990).

[4] H.A. Bethe, Ann. Phys. (Leipzig) 5, 325 (1930).

[5] F. Bloch, Ann. Phys. (Leipzig) 16, 285 (1933).

[6] O.B. Firsov, Zh. Eksp. Teor. Fiz. 36, 1517 (1959) [Sov. Phys.-JETP 9, 1076 (1959)].

[7] P. Sigmund, Phys. Rev. A 26, 2497 (1982).

[8] J. Lindhard, A. Winter, K. Dan. Vidensk. Selsk. Mat.-Fys. Medd. 34, no. 4 (1964).

[9] R.H. Ritchie, Phys. Rev. 114, 644 (1959).

[10] P.M. Echenique, R.M. Nieminen, R.H. Ritchie, Solid State Commun. 37, 779 (1986).

[11] D.R. Bates, W. Griffing, Proc. Phys. Soc. A 66, 961 (1953); 67, 663 (1954); 68, 90 (1955).

[12] W. Brandt, M. Kitagawa, Phys. Rev. B 25, 5631 (1982).

[13] Y.K. Kim, K. Cheng, Phys. Rev. A 22, 61 (1980).

[14] R. Cabrera-Trujillo, S.A. Cruz, J. Oddershede, J.R. Sabin, Phys. Rev. A 55, 2864 (1997).

[15] D.R. Bates, in: Atomic and Molecular Processes, Ed. D.R. Bates, Academic Press, New York 1962, ch. 14.

[16] R.W. James, in: The Optical Principles of the Diffraction of X-rays, Ed. L. Brag, Bell, London 1967, p. 109.

[17] H.A. Bethe, R. Jackiw, Intermediate Quantum Mechanics, Benjamin/Cummings, Reading (MA) 1986, p. 393.

[18] N. Bohr, Phys. Rev. 58, 654 (1940); 59, 270 (1941).

[19] B.S. Yarlagadda, J.E. Robinsin, W. Brandt, Phys. Rev. B 17, 3473 (1978).

[20] L.H. Thomas, Proc. Cambridge Philos. Soc. 23, 542 (1927); E. Fermi, Mem. Accad. Lincei 6, 602 (1927).

[21] J.F. Ziegler, J.P. Biersack, U. Littmark, The Stopping and Range of Ions in Solids, Pergamon, New York 1985, p. 321.

[22] M.A. Kumakhov, F.F. Komarov, Energy Loss and Ions Ranges in Solids, Gordon and Breach, New York 1981, p. 296.

[23] H. Sugiyama, J. Phys. Soc. Jpn. 41, 1339 (1976).

[24] J. Lindhard, M. Scharff, Dan. Mat. Fys. Medd. 27, 1 (1953).

[25] H.D. Betz, G. Hortig, E. Leischner, Ch. Schmelzer, B. Stadler, J. Weihrauch, Phys. Lett. 22, 643 (1996).

[26] J.M. Anthony, W.A. Lanford, Phys. Rev. A 22, 1868 (1982).

[27] J.H. Hubbel, W.J. Veigele, E.A. Briggs, R.T. Brown, D.T. Cromer, R.J. Howerton, J. Phys. Chem. Ref. Data 4, 471 (1975). 
[28] H. Paul, A. Schinner, At. Data Nucl. Data Tables 85, 377 (2003).

[29] J.F. Ziegler, J.P. Biersack, in: SRIM-2003. The Stopping and Range of Ions in Matter, www.srim.org/SRIM. 